\title{
A importância da resina composta Bulk Fill na odontologia moderna
}

\author{
The importance of Bulk Fill compound resin in modern dentistry \\ La importancia de la resina compuesta Bulk Fill en la odontología moderna \\ Déborah Cristina Fernandes David Siqueira Sandes ${ }^{1 *}$, Izabel Cristina Gomes de Mendonça.
}

\begin{abstract}
RESUMO
Objetivo: Abordar por meio de uma revisão narrativa os fundamentais pontos clínicos das Resinas Compostas Bulk Fill (RCBF) e sua importância no consultório odontológico. Revisão bibliográfica: Com o intuito de preencher algumas características não desejáveis presentes na RC, visando aprimorar e melhorar a durabilidade além do tempo clínico para sua implantação, foram realizadas inúmeras pesquisas com o objetivo de melhorar esses fatores. Uma dessas principais alterações feitas foi realizada em seu componente orgânico, tanto na quantidade estabelecida quanto em sua composição, continuando sem alterações sua matriz principal, que são polímeros à base de metacrilato. Assim sendo, foram estabelecidas as resinas compostas Bulk Fill, em que viabilizaram ao Cirurgião Dentista $(C D)$ a sua aplicação de maneira única dentro da restauração do elemento dental. Considerações finais: A aplicação das RCBF simplifica o dia a dia do $\mathrm{CD}$ por restringir de maneira considerável inúmeros passos e estágios que as RC demandam, as RCBF indicam certo equilíbrio de coloração. O uso das resinas Bulk Fill concede que se deixe de lado a técnica tradicional de incrementação, reduzindo-se o tempo do paciente no consultório.
\end{abstract}

Palavras-chave: Estética, Polimerização, Força compressiva.

\begin{abstract}
Objective: To approach through a narrative review of the fundamental clinical points of Bulk Fill Composite Resins (RCBF) and their importance in the dental office. Bibliographic review: In order to fill some undesirable characteristics present in CR, to improve and improve durability in addition to the clinical time for its implantation, researches were completed with the objective of these factors. One of these main changes was made in its organic component, both in the desired quantity and in its composition, without changing its main matrix, which are polymers based on methacrylate. Therefore, Bulk Fill composite resins were made, in which the Dental Surgeon (CD) made it possible to apply them in a unique way within the restoration of the dental element. Final considerations: The application of the RCBF simplifies the day-to-day of the DC by restricting in a way, the steps and stages that the CR require, since the RCBF indicates a certain balance of color. The use of Bulk Fill resins allows to leave aside the traditional technique of increment, if the time of the patient in the office.
\end{abstract}

Keywords: Esthetics, Polymerization, Compressive strength.

\section{RESUMEN}

Objetivo: Abordar mediante una revisión narrativa los puntos clínicos fundamentales de las Resinas Compuestas Bulk Fill (RCBF) y su importancia en el consultorio odontológico. Revisión bibliográfica: Con el fin de llenar algunas características indeseables presentes en la RC, mejorar y mejorar la durabilidad más allá del tiempo clínico para su implantación, se completaron investigaciones con el objetivo de estos factores. Uno de estos principales cambios se realizó en su componente orgánico, tanto en la cantidad deseada como en su composición, sin cambiar su matriz principal, que son polímeros a base de metacrilato. Por ello, se elaboraron las resinas compuestas Bulk Fill, en las que hicieron posible que el Cirujano Dentista (CD) las aplicara de forma única dentro de la restauración del elemento dental. Consideraciones finales: La aplicación del RCBF simplifica el día a día del DC al restringir de alguna manera, los pasos y etapas que requiere el $\mathrm{RC}$, ya que el RCBF indica un cierto equilibrio de color. El uso de resinas Bulk Fill permite dejar de lado la técnica tradicional de incremento, si el tiempo del paciente en el consultorio.

Palabras clave: Estética, Polimerizacion, Fuerza compresiva.

${ }^{1}$ Centro Universitário CESMAC (CESMAC), Maceió - AL. *E-mail: deborahcfdsiqueira@hotmail.com

SUBMETIDO EM: $3 / 2021$

ACEITO EM: 3/2021

PUBLICADO EM: $3 / 2021$ 


\section{INTRODUÇÃO}

Nas últimas décadas a odontologia reabilitadora dos quais utilizam a técnica adesiva vem ocupando uma área de suma importância não apenas ao que tange procedimentos estéticos como também na parte funcional e mecânica dos elementos dentais anteriores e posteriores, no início dos estudos dos quais buscavam a reabilitação dos dentes utilizava-se muito o amálgama de prata, que apresentava uma liga metálica em que um dos metais envolvidos apresenta-se em estado líquido, sendo geralmente o mercúrio, com seu desuso devido a presenta de substâncias cancerígenas presentes no mercúrio parou de ser utilizado dando lugar as resinas compostas. Contudo mesmo sabendo boa sua boa utilização, as resinas apresentam problemas devido sua durabilidade principalmente nos dentes posteriores (CAVALHEIRO JP, et al., 2014).

Os compósitos dos quais apresentam como finalidade a união de outros materiais com o objetivo de se obter um produto de maior qualidade, são utilizados nos consultórios odontológicos por causa de sua forma adequada ao que se refere à adesividade, além de dispor de uma boa adequação de adaptar-se aos tecidos presentes nos elementos dentais (ALKHUDHAIRY FI, 2017). No entanto, o intervalo de tempo clínico que precisa para fazer sua inserção e sua rigidez de maneira técnica são contestadores. Um do fator principal que é atribuído a técnica de utilização de resina composta $(R C)$ tradicional é relacionado a sua forma incremental, em que se refere na inserção de RC colocando-se no preparo pequenas quantidades de resinas e ir esculpindo e fotopolimerizando até obter o resultado final esperado (ALMEIDA LJDS, et al., 2017).

Com o intuito de preencher algumas características não desejáveis presentes na $\mathrm{RC}$, visando aprimorar e melhorar a durabilidade além do tempo clínico para sua implantação, foram realizadas inúmeras pesquisas com o objetivo de melhorar esses fatores. Uma dessas principais alterações feitas foi realizada em seu componente orgânico, tanto na quantidade estabelecida quanto em sua composição, continuando sem alterações sua matriz principal, que são polímeros à base de metacrilato (ALSHALI RZ, et al., 2013).

Assim sendo, foram estabelecidas as resinas compostas Bulk Fill, em que viabilizaram ao Cirurgião Dentista $(C D)$ a sua aplicação de maneira única dentro da restauração do elemento dental. Esta nova técnica significou muito para a odontologia reabilitadora, minimizando o tempo clinico que os profissionais utilizavam nas técnicas utilizadas à anos atrás. Em decorrência, o processo restaurador ficou mais agradável tal para o paciente quanto para o CD, em que seu trabalho foi otimizado com o tempo em que o paciente passa em um tratamento. Os princípios ativos presentes nesses materiais, como sua fluidez, por ser translucido, apresenta pequeno módulo de elasticidade e baixa constrição de polimerização, faz com que as margens presentes na cavidade dentária sejam fixadas com maior precisão, sem grande risco de ocorrer processos de infiltração, ajudando com isso a durabilidade do tratamento realizado (KIM RJY, et al., 2015).

Este artigo apresenta como principal objetivo, revisar e demostrar as inúmeras vantagens da utilização no consultório das resinas Bulk Fill em comparação com às resinas compostas convencionais.

\section{REVISÃO BIBLIOGRÁFICA}

\section{Sistema adesivo}

A utilização do sistema adesivo na odontologia restauradora apresenta um papel fundamental, visto que eles são utilizados para que ocorra o processo de junção entre o material restaurador às estruturas presentes nos elementos dentais. Apresentam uma combinação de princípios ativos dos quais faz-se presente os monômeros resinosos hidrofóbicos de distinto peso molecular e viscosidade, solventes resinosos e diluentes orgânicos, como é o caso da acetona, álcool ou água (GUIMARÃES LF, et al., 2013).

São utilizadas nos processos restauradores de maneira estética acometida por lesões cariosas, anatomia indesejada do elemento dental, coloração ou pigmentação dos elementos dentais, na fixação de fragmentos através da colagem, no preparo de restaurações indiretas, podendo ser utilizado também como selamento de fóssulas e fissuras, na implantação de braquetes na área ortodôntica, na reparação de restaurações defeituosas, na criação de um núcleo para receber coroas, no processo de cimentação de pinos intraradiculares e para retirada de sensibilidade nas raízes expostas principalmente em dentes anteriores (CANEPPELE T e BRESCIANI E, 2016). 
Estudos pioneiros ao que se refere a técnica de condicionamento e adesão no esmalte dental foi realizado pelo pesquisador Buonocore no ano de 1955, que sugestionou, realizar condicionamento na parte mais superficial do esmalte dental utilizando ácido (DIJKEN JWV, et al., 2015). Para que ocorra a adesão entre as estruturas do dente e a resina é necessário a utilização de algumas substâncias como é o caso do ácido, solventes e monômeros, visto que eles possuem força através dos seus princípios ativos para modificarem as estruturas fisiológicas do esmalte dental como da dentina (EL-DAMANHOURY H e PLATT J, 2014).

\section{Característica e propriedade da RC}

No início a RC utilizada, era a macropartículada. Sendo categorizada assim por causa do comprimento das suas partículas de carga inorgânica que exibiam. As proporções das partículas são estabelecidas entre 10 a 100 micrómetro $(\mu \mathrm{m})$, o que retrata uma percentagem de $60 \%$ da quantidade total da RC (ELSHARKASI $M$, et al., 2015). No entanto, exibiam grande espessura na parte mais superficial, e passavam por uma deterioração de maneira abrasiva em sua matriz. Desse modo, as partículas que possuíam carga com maior dureza tornavam-se evidenciadas, ocasionando em sua superfície um acabamento mais áspero e ocasionando por sua vez maior prevalência de manchas no elemento dental restabelecido, além de sua durabilidade ser reduzida (FERRACANE JL, 2011).

De forma estrutural, as RC's apresentam em sua constituição as seguintes etapas: matriz orgânica que é a parte responsável pela resistência, rigidez e estabilidade , carga inorgânica em que confere rigidez na parte mais superficial e melhor resistência à compressão e tração, elevando a sua durabilidade e o desempenho clínico; diminuindo a contração de polimerização e a contração e expansão térmica; controla a viscosidade e as características de manipulação e o silano que é um agente de união e sendo indicado para estabelecer a união química entre materiais orgânicos, adesivos contendo monômeros metacrílicos e materiais inorgânicos (FERREIRA AB e SILVA NEF, 2017).

Sua matriz orgânica é constituída por bisfenol glicidil metacrilato (Bis GMA) ou poliuretano que é um polímero que compreende uma cadeia de unidades orgânicas unidas por ligações uretânicas. Devido ao fato desses compósitos exibirem uma grande viscosidade, foram acrescentados monómeros que apresentam pequena viscosidade, o trietilenoglicol dimetacrilato (TEGMA) e o etilenoglicol dimetacrilato (EDGMA), devido ao fato de possuir maior flexibilidade. A sua composição e a maneira versátil da matriz orgânica presente, possui intervenções na hora da polimerização, no nível e modificação de monómeros em polímeros que são macromoléculas estabelecidas a partir de unidades estruturais menores, em sua viscosidade e no absorvimento de água (MELO J, et al., 2011; FERREIRA AB e SILVA NEF, 2017).

Sua matriz inorgânica é composta por partículas de carga, em que um dos seus principais objetivos é de aumentar a durabilidade através da resistência no ato da tração mecânica do material, podendo influenciar de maneira direta em inúmeras propriedades, por exemplo, na dureza, o grau de amplificação térmica, a absorção de água, a luminosidade do elemento dental trabalhado, o seu módulo de elasticidade e a resistência a possíveis fraturas (FESTUCCIA MS, et al., 2012).

O silano é de suma importância no processo restaurador, através dele a matriz orgânica presente não se solta da carga inorgânica, precautelando possíveis aparecimentos de bolhas de ar (local onde a água pode ser armazenada) que reduz o problema na parte mecânica das restaurações feitas (EL-DAMANHOURY H e PLATT J, 2014).

A RC micropartículada, foram produzidas com a intuito de aprimorar as características das resinas compostas macroparticuladas. Suas partículas de carga presentes são formadas por sílica coloidal ou sílica amorfa, cuja sua espessura possui uma variação de 0,02 a $0,1 \mu \mathrm{m}$, integrando de $20 \%$ a $60 \%$ da porção total da RC. Por decorrência de sua constituição, as propriedades mecânicas são consideradas pequenas, estabelecendo-se assim fatores limitantes, em que as RC's micropartículadas sejam utilizadas para tratamento restauradores de elementos dentais que suportam pouco impacto na hora da mastigação (GUIMARÃES LF, et al., 2013).

Para a obtenção do equilíbrio referente aos proveitos específicos de maneira particularizada de cada grupo, foram inseridas as RC's microhíbridas, dos quais foram estabelecidos através da mistura da 
constituição micropartículada e macropartículada, agregando dessa maneira, as características das duas classes de partículas incorporadas. Expondo, em maior número de casos, de $10 \%$ a $20 \%$ na porção de micropartículas de sílica coloidal e de 50\% a 60\% de macropartículas de vidro de metais consistente. Estas RC's exibem uma porcentagem de carga que coincide entre $75 \%$ a $80 \%$ do seu peso molecular. $O$ que distingui uma RC microhíbrida de uma híbrida é a sua superior dimensão de resinas compostas micropartículas no que diz respeito às macropartículas (ALSHALI RZ, et al., 2013; GUIMARÃES LF, et al., 2013).

A procura de um material que apresentem partículas de carga gradativamente menores, e que concedesse a inclusão de um grande acúmulo de carga à matriz orgânica, procederam no estabelecimento das RC's nanopartículadas. Esse grupo de resinas compostas apresentam partículas com tamanhos nanométricos. Devendo serem consideradas como resinas compostas nanopartículadas, aquelas que exibem partículas com tamanhos que compreendem entre 1 a $10 \mathrm{~nm}$ (nanometro) (ALMEIDA LJDS, et al., 2017).

Para que a RC obtenha suas características de forma ideal, é de suma importância que se leve em atenção seu grau de transformação. O grau de transformação para que ocorra a conversão reproduz as dimensões de monômeros resinosos que são sensibilizados na hora da sua polimerização e mudados em polímeros. Tem-se levado em consideração que o grau de conversão habitualmente alcançado pelas RC's fica à volta de $60 \%$. Os princípios que influenciam no grau de conversão de modificação são os tipos de fotopolimerizadores utilizados, a distância que o profissional utiliza o fotopolimerizador e o compósito resinoso, o comprimento da quantidade de resina composta introduzida na cavidade preparada pelo CD, a forma de opacidade da resina estabelecida, entre outros fatores. Quanto mais perto for a fonte de luz no preparo restaurador, maior será a parcela de monômeros que sofrerão polimerizados (ALKHUDHAIRY FI, 2017).

A utilização da técnica de forma incremental é um método que consiste na habilidade de fazer a manipulação do material trabalhado pelo Cirurgião Dentista, melhor dizendo, o êxito do processo restaurador é sensível à capacidade do executor sobre a RC. É fundamental uma precisa disposição do material, de maneira especial nas margens que circundam a cavidade, de maneira a diminuir as tensões instituída pela contração na hora da polimerização (CAVALHEIRO JP, et al., 2014; ELSHARKASI M, et al., 2015).

Desse modo, são efetuadas inserções com pequenos incrementos, devido a delimitada profundidade de se estabelecer uma polimerização satisfatória. A densidade dos incrementos colocados na cavidade não deve exceder mais que $2 \mathrm{~mm}$ (FLURY S, et al., 2014).

\section{Resinas Bulk Fill}

Em preparos cavitários em que apresentam cavidades muito fundas e expandidas, a metodologia do processo incremental de inserção das RC's exige um maior tempo de trabalho do operador, sendo aumentado possíveis riscos de ocorrer contaminação no meio trabalhado por fluidos presentes na cavidade oral, além de ser mais fácil o surgimento de bolhas de ar entre os incrementos colocados. Levando em consideração esses fatores de limitações e deficiências correlacionadas às RC's de utilização direta, os fabricantes desses materiais restauradores vêm investindo crescentemente mais na idealização de um material que preencha a maior parte dos requisitos físicos, mecânicos e estéticos imprescindíveis para obtenção de um material restaurador melhor, que possuam as atribuições garantidas na efetuação de restaurações mais satisfatórias e com oportuno comportamento clínico (GONÇALVES F, et al., 2018).

Nos últimos anos foram observados que os pacientes ficaram mais exigentes e passaram a querer resultados mais rápidos com uma qualidade maior, esses fatores fizeram com quer os fabricantes de materiais odontológicos tivessem que desenvolver e estabelecer um novo grupo na categoria de RC's à base de metacrilato, surgindo assim as resinas Bulk Fill. Para os pacientes, essa nova classe significou maior comodidade e, para os Cirurgiões Dentistas, a atenuação do tempo de trabalho. As resinas Compostas do tipo bulk fill (RCBF) proporcionaram a diminuição do tempo de fotopolimerização e da porção de incrementos essenciais. Além de obter uma fluidez melhor e permite sua aplicação de até $5 \mathrm{~mm}$ de espessura sem que ocorra grande contração na hora da polimerização, além do que, exibem uma grande resposta à fotoativação pela luz, conferida por meio da evolução do sistema fotoiniciador (GONÇALVES F, et al., 2018; KAYA MS, et al., 2018). 


\section{Características das resinas Bulk Fill}

Os componentes presentes na RCBF são estabelecidos pela incorporação de uma matriz orgânica, partículas de carga, por moléculas precursoras de polimerização e do silano que é o agente de união, dos quais estabelecem uma interligação entre a matriz orgânica e suas partículas de carga (GONÇALVES F, et al., 2018). Apresentam pequenas tensões, quando são relacionadas à diminuição de polimerização, e apresentam excelentes características ao que se refere a transmissão de luz, por causa da diminuição da dispersão da luz na junção entre as matrizes de partículas inorgânicas, além de possuir uma boa forma de resistência na hora da união, sem depender de forma direta da estrutura do preparo cavitário e da técnica de implementação (KEMALOGLU H, et al., 2015).

As RCBF podem serem constituídas através de duas formulações distintas que estão relativas à sua forma de consistência, podendo ser de baixa viscosidade (flow), ou de alta viscosidade (KEMALOGLU H, et al., 2015).

A flow possui sua indicação como sendo um material restaurador de base e precisa que o CD faça um incremento de $2 \mathrm{~mm}$ de RC convencional seja incorporado sobre uma das suas camadas, para obter uma maior garantir em sua resistência ao problema de desgaste, pois exibe uma baixa dureza na parte superficial devido a menor quantidade de cargas inorgânicas presentes em sua constituição (KEMALOGLU H, et al., 2015).

As resinas Bulk Fill que apresentam grande viscosidade, apresentam um potencial de em inseridas na cavidade de forma única, sem precisar de incrementações, em toda área da cavidade dentaria onde será efetuada o preparo restaurador (SILVA NETO JM, et al., 2019).

Experimentos realizados através de pesquisas têm qualificado as características mecânicas das RCBF quando introduzidas na cavidade do elemento dental em incrementos de $4 \mathrm{~mm}$ à $5 \mathrm{~mm}$ de profundidade. Com isso cada vez mais vem se estabelecendo com clareza que, a despeito do maior volume inserido quando se compara as resinas compostas tradicionais, a RCBF apresentam baixa constrição na hora e sua polimerização (HIRATA R, et al., 2015; SILVA NETO JM, et al., 2019).

Com o objetivo de obter um maior aumento na profundidade da cavidade sem perde os resultados positivos na hora da polimerização, os fabricantes das RCBF transformaram a translucidez e a opacidade dos componentes presentes e reduziram a quantidade de partículas inorgânicas, isso porque a entrada da luz encontra-se profundamente correlacionada com a porção de partículas que estão presentes. Para estabelecer apropriada transformação de monômeros em polímeros, mesmo se complementando os incrementos de $5 \mathrm{~mm}$, para isso foram implantados fotoiniciadores com maior impregnação de luz (GONÇALVES F, et al., 2018; KAYA MS, et al., 2018).

É essencial evidenciar que, para poder atingir a fotoativação das resinas Bulk Fill na profundidade estabelecida pelos fabricantes, têm que ser utilizados fotopolimerizadores de apresentem ótima propriedades, com um potencial mínimo de 800 Megawatt por centímetro cubico $(\mathrm{mW} / \mathrm{cm} 2)$, sendo o mais correto utilizar os que apresentam uma potência de $1000 \mathrm{~mW} / \mathrm{cm} 2$, já que uma parcela da luz é perdida antes de chegar nas camadas que estão nas partes mais profundas da restauração (YSSA I, et al., 2016).

Já que a polimerização das RC's está de forma direta correlacionada às suas características físicoquímicas, essas particularidades das RCBF, vêm se tornado com frequência motivo de inúmeros estudos. Mesmo assim, ainda encontram-se algumas imprecisões associadas com suas propriedades térmicas, sua força de resistência à mastigação e de contração na hora da polimerização, sendo indispensável ainda a execução de investigações científicas fundamentadas em estudos clínicos reunidos a um longo prazo de tempo (KAYA MS, et al., 2018).

\section{Aplicação Clínica}

Hirata R, et al. (2015) apresentaram duas técnicas no emprego clínico das RCBF, sendo uma das técnicas estabelecida através de dois passos denominada "Técnica de escultura semelhante a amálgama em duas etapas" e uma outra técnica que é estabelecido em uma única etapa, denominada "técnica de esculpir tipo 
amálgama de um único passo". Na utilização da Técnica de escultura semelhante a amálgama em duas etapas, primeiramente coloca-se uma resina Bulk Fill com pequena viscosidade e, logo depois, utiliza-se uma camada de resina Bulk Fill com grande viscosidade. Na parte em que é implementado a resina de grande viscosidade é feito a escultura, estabelecendo dessa maneira a anatomia do elemento dental. Enquanto a técnica que confere um único passo é utilizada unicamente a resina Bulk Fill de alta viscosidade. Com essa forma de utilizar em apenas um passo, o profissional pode colocar até $5 \mathrm{~mm}$ de resina do tipo Bulk Fill de uma única vez, certificando-se de uma boa polimerização de maneira eficaz. Tornando assim as consultas e 0 tratamento terapêutico menos demorado, dando um melhor conforto ao paciente.

Quando comparada a resina composta as resinas Bulk Fill dispõe de maior translucidez, esse fator permite com quer a luz do fotopolimerizador penetre com maior facilidade até a última camada trabalhada. Além disso, elas apresentam um superior escoamento, garantindo assim a incorporação nas áreas e ângulos que apresentam dificuldades de serem feitos (TAUBOCK T, et al., 2018).

Ilie N e Stark K (2014), examinaram algumas marcas comerciais da RCBF, em sua pesquisa foi analisado a profundidade de polimerização em sua aplicação no consultório. Oscilando e levando em consideração o tempo e a potência utilizada no fotopolimerizador, podendo chegar a concluírem que, para obtenção de um resultado satisfatório com os compósitos Bulk Fill, o tempo de fotopolimerização deve ser de 20 segundos, tendo capacidade de polimerizar cavidades de até $5 \mathrm{~mm}$.

Dijken JWV, et al. (2015), analisaram restaurações efetuadas em preparos cavitários de classe I, dos molares permanentes. Nessa pesquisa fizeram um grau comparativo das RCBF com as RC's fluídas normais. Para tal fim, aplicaram nas cavidades RCBF com incrementação única de $5 \mathrm{~mm}$ e logo em seguida fotopolimerizaram, de acordo com as recomendações do fabricante. Da mesma forma utilizaram a RC, no entanto a forma de introduzir foi incremental. O resultado estabelecido foi parecido, todavia, as RCBF demonstraram otimização do tempo no consultório.

Almeida LJDS, et al. (2017), efetivaram uma pesquisa referente ao poder de união entre as estruturas dos elementos dentários e as RCBF. Nessa pesquisa analisou-se cinquenta molares humanos com preparos restauradores de classe I. Após a efetuação das restaurações, os elementos dentais passaram por secção e colocados em exames que comprovaram que as RCBF mostraram uma força adesiva superior às utilizadas no uso das RC's.

\section{CONSIDERAÇÕES FINAIS}

Com embasamento na literatura realizada, verifica-se que, na maioria dos casos, as RCBF apresentam características semelhantes com as RC's, quando é utilizado o protocolo de procedimento padrão de cada uma delas. A aplicação das RCBF simplifica o dia a dia do $C D$ por restringir de maneira considerável inúmeros passos e estágios que as RC demandam, as RCBF indicam certo equilíbrio de coloração. O uso das resinas Bulk Fill concede que se deixe de lado a técnica tradicional de incrementação, reduzindo-se o tempo do paciente no consultório, elas estão presentes no mercado há poucos anos, com isso é necessário a efetuação de mais pesquisas cientificas, além do rastreamento clínico das restaurações executadas, para só assim poder ser assegurado de maneira concreta o sucesso clínico e a durabilidade do processo terapêutico realizado.

\section{REFERÊNCIAS}

1. ALKHUDHAIRY FI. The effect of curing intensity on mechanical properties of different bulk-fill composite resins. Clin Cosmet Investig Dent, Kingdom of Saudi Arabia, 2017; 1-6.

2. ALMEIDA LJDS, et al. Is there correlation between polymerization shrinkage, gap formation, and void in bulk fill composites? A $\mu$ CT study. Brazilian Oral Research, 2017; 31:1-10.

3. ALSHALI RZ, et al. Degree of conversion of bulk-fill compared to conventional resin-composites at two time intervals. Dental Materials, 2013; 29(9): 213-217.

4. CANEPPELE T, BRESCIANI E. Resinas bulk-fill - O estado da arte. Revisão de literatura. Departamento de Odontologia Restauradora do Instituto de Ciência e Tecnologia do Campus de São José dos Campos - Unesp, 2016.

5. CAVALHEIRO JP, et al. Marginal adaptation of class V restorations with current-generation dentin-bonding agents:effect of different dentin surface treatments. J Contemp Dent Pract, 2014; 15(3): 331-335. 
6. DIJKEN JWV, et al. Randomized 3-year clinical evaluation of class I and II posterior resin restorations placed with a bulk fill resin composite and a one-step self-etching adhesive. J Adhes Dent, New Malden, 2015; 17: 81-88.

7. EL-DAMANHOURY H, PLATT J. Polymerization shrinkage stress kinetics and related properties of bulk-fill resin composites. Oper Dent, 2014; 39(4): 374-382.

8. ELSHARKASI M, et al. Cuspal deflection in premolar teeth restored with bulk-fill resinbased composite materials. Tese (Master of Science in Dentistry) - Indiana University School of Dentistry, Indiana, 2015.

9. FERRACANE JL. Resin composite--state of the art. Dent Mater, Portland, 2011; 27(1): 29-38.

10. FERREIRA AB, SILVA NEF. Utilização das Resinas Compostas Bulk Fill: uma revisão da literatura. 2017. Trabalho de conclusão de curso (Bacharel em Odontologia) - Faculdade Integrada de Pernambuco, Pernambuco, 2017.

11. FESTUCCIA MS, et al. Pires-de- Souza F de C. Color stability, surface roughness and microhardness of composites submitted to mouthrinsing action. J Appl Oral Sci. 2012; 20(2): 200-205.

12. FLURY S, et al. Influence of increment thickness on microhardness and dentin bond strength of bulk fill resin composites. Dent Mater, Bern, 2014; 30(10): 1104-12.

13. GONÇALVES F, et al. A comparative study of bulk-fill composites: degree of conversion, post-gel shrinkage and cytotoxicity. Braz Oral Res, São Paulo, 2018; 32.

14. GUIMARÃES LF, et al. Silanização de partículas de carga de Compósitos Odontológicos - Revisão De Literatura. Revista Da Faculdade de Odontologia, 2013; 18(2): 254-260.

15. HIRATA R, et al. Bulk Fill Composites: An Anatomic Sculpting Technique. Journal of Esthetic and Restorative Dentistry, $2015 ; 27(6): 335-343$.

16. ILIE N, STARK K. Curing behavior of high-viscosity bulk-fill composites. J Dent, 2014; 42(8): 977-985.

17. KAYA MS, et al. Structural and mechanical properties of a giomer-based bulk fill restorative in different curing conditions. J Appl Oral Sci, Turkey, 2018.

18. KEMALOGLU $\mathrm{H}$, et al. Effect of novel restoration techniques on the fracture resistance of teeth treated endodontically: an in vitro study. Dental Material Journal, Washington, 2015; 34 (5): 618-622.

19. KIM RJY, et al. Polymerization shrinkage, modulus, and shrinkage stress related to tooth-restoration interfacial debonding in bulk-fill composites. Journal of Dentistry, 2015; 43(4): 430-439.

20. MELO J, et al. Selecionando corretamente as resinas compostas. Int J Dent. 2011; 10(2): 91-96.

21. SILVA NETO JM, et al. O uso das resinas compostas tipo bulk fill: Uma revisão de literatura. Revista Eletrônica Acervo Saúde, 2019; 37: 1-8.

22. TAUBOCK T, et al. Polymerization shrinkage and shrinkage force kinetics of high- and low-viscosity dimethacrylateand ormocer-based bulk-fill resin composites, Odontology, Zurich, 2018.

23. YSSA I, et al. Effect of curing light emission spectrum on the nano hardness and elastic modulus of two bulk-fill resin composites. Dental Materials, 2016; 32(4): 535- 550. 